\title{
Classical molecular dynamics simulation of weakly-bound projectile heavy-ion reactions
}

\author{
Mitul R. Morker ${ }^{1}$ and Subodh S. Godre ${ }^{1, a}$ \\ ${ }^{1}$ Department of Physics, Veer Narmad South Gujarat University, Surat 395007, India
}

\begin{abstract}
A 3-body classical molecular dynamics approach for heavy-ion reactions involving weakly bound projectiles is developed. In this approach a weakly bound projectile is constructed as a two-body cluster of the constituent tightly bound nuclei in a configuration corresponding to the observed breakup energy. This 3-body system with their individual nucleon configuration in their ground state is dynamically evolved for given initial conditions using the three-stage classical molecular dynamics approach (3S-CMD). Various levels of rigidbody constraints on the projectile constituents and the target are considered at appropriate stages. This 3dimensional approach explicitly takes into account not only the long range Coulomb reorientation of the deformed collision partner but internal excitations and breakup probabilities at distances close to the barrier also. Dynamical simulations of ${ }^{6} \mathrm{Li}+{ }^{209} \mathrm{Bi}$ show all the possible reaction mechanism like complete fusion, incomplete fusion, scattering and breakup scattering. Complete fusion cross sections of ${ }^{6} \mathrm{Li}+{ }^{209} \mathrm{Bi}$ and ${ }^{7} \mathrm{Li}+{ }^{209} \mathrm{Bi}$ reactions are calculated in this approach with systematic relaxations of the rigid-body constraints on one or more constituent nuclei.
\end{abstract}

\section{Introduction}

Heavy-ion fusion involving weakly bound but stable nuclei are affected by their low binding energy, which can cause them to breakup near the fusion barrier [1-4]. If all the projectile fragments are captured by the target nucleus then it is termed as complete fusion (CF), irrespective of whether they break-up (SCF-sequential complete fusion) or do not breakup (DCF-direct complete fusion) before being captured. However, if only some of the fragments are captured then it is termed as an incomplete fusion (ICF). Such ICF processes can lead to suppression of fusion probabilities. In a recent experiment it is observed that direct processes such as nucleon transfer reactions leading to breakup of the remaining projectile can also contribute significantly to the ICF processes [4].

Breakup reactions have been studied using macroscopic approaches such as Continuum Discretized Couple-Channel (CDCC) method [5] and a semi-classical couple channel approximation [6]. A classical trajectory model $[7,8]$ also has been used to study fusion of weakly bound nuclei. In the later model the projectile is considered as a two-body system which interacts with each other by an assumed weak potential and the breakup is initiated by a breakup probability function. However, deformation and consequential reorientation of the entire projectile system is neglected in this model. Moreover, the interaction potentials are not obtained self- consistently. None of the above models account for breakup following direct reactions in the ICF processes.

It is shown using the Classical Rigid-Body Dynamics (CRBD) calculations that reorientation of a light deformed nucleus arising from the long range Coulomb torques has significant effect on the fusion process [9]. Similarly, using a 3-stage Classical Molecular Dynamics (3S-CMD) approach it is shown that internal excitations of the nuclei at very close distances also has significant effect on the fusion process $[10,11]$.

A classical trajectories model is developed which is an extension of the $3 \mathrm{~S}-\mathrm{CMD}$ model. In this approach a weakly bound nucleus is constructed as a cluster of two or more tightly bound nuclei consisting of point nucleons. The interaction between the projectile fragments is generated self-consistently. Both the projectile and the target are extended objects with desired size and shape deformation. Relaxation of the rigid-body constraints at appropriate stages in the dynamical simulation takes care of the possible excitations of target and projectile fragments which may also lead to projectile breakup.

The details of the model calculation are given in section-2. Dynamical simulations of ${ }^{6} \mathrm{Li}+{ }^{209} \mathrm{Bi}$ at a given collision energy and different impact parameters are discussed in section-3. Complete fusion cross-section calculations with systematic relaxation of rigid-body constraints on one or more nuclei of ${ }^{6} \mathrm{Li}+{ }^{209} \mathrm{Bi}$ reaction are given in section-4.1 and those for ${ }^{7} \mathrm{Li}+{ }^{209} \mathrm{Bi}$ are given in section-4.2. Finally conclusions are given in section-5.

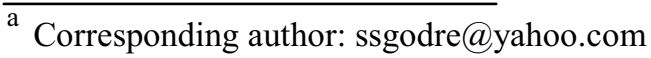




\section{Calculation details}

\subsection{Construction of nuclei}

Nucleon distribution in each tightly bound nucleus is first obtained by the STATIC code [12] in which the total potential energy of an initially random distribution of nucleons is cyclically minimized. Nucleons are assumed to be classical point particles without spin which interact via suitable two-body forces. The Coulomb potential between protons has the usual form

$$
V_{c}\left(r_{i j}\right)=\frac{1.44}{r_{i j}} \mathrm{MeV}
$$

and the NN potential chosen is a soft-core Gaussian potential of the form [12]

$$
V_{N}\left(r_{i j}\right)=-V_{0}\left(1-\frac{C}{r_{i j}}\right) \exp \left(-\frac{r_{i j}^{2}}{r_{0}^{2}}\right)
$$

The NN potential between like particles is taken to be $20 \%$ weaker than that between unlike particles [13].

A parameter set $V_{O}=710 \mathrm{MeV}, C=1.88 \mathrm{fm}, r_{O}=1.15$ $\mathrm{fm}$ is used which closely reproduces the ground-state properties of ${ }^{209} \mathrm{Bi}$. Ground-state properties of the other tightly bound nuclei, ${ }^{2} \mathrm{H}(d),{ }^{4} \mathrm{He}(\alpha)$ and ${ }^{3} \mathrm{H}(t)$ generated with the above potential are given in the Table-1.

Table-1: Ground-state properties of the constructed nuclei.

\begin{tabular}{|c|r|r|r|r|}
\hline & \multicolumn{2}{|c|}{ Calculated } & \multicolumn{2}{c|}{ Experimental } \\
\cline { 2 - 5 } & $\begin{array}{c}\text { B.E. } \\
(\mathbf{M e V})\end{array}$ & $\begin{array}{c}\mathbf{R} \\
(\mathbf{f m})\end{array}$ & $\begin{array}{c}\text { B.E. } \\
(\mathbf{M e V})\end{array}$ & $\begin{array}{c}\mathbf{R} \\
(\mathbf{f m})\end{array}$ \\
\hline${ }^{\mathbf{2}} \mathbf{H}(\boldsymbol{d})$ & 2.07 & 1.00 & 2.23 & 2.10 \\
\hline${ }^{4} \mathbf{H e}(\boldsymbol{\alpha})$ & 14.48 & 1.32 & 28.30 & 1.69 \\
\hline${ }^{3} \mathbf{H}(\boldsymbol{t})$ & 7.58 & 1.24 & 8.48 & 1.68 \\
\hline${ }^{\mathbf{6}} \mathbf{L i}(\boldsymbol{\alpha}+\boldsymbol{d})$ & 18.02 & 1.93 & 31.99 & 2.54 \\
\hline${ }^{7} \mathbf{L i}(\boldsymbol{\alpha}+\boldsymbol{t})$ & 24.53 & 2.06 & 39.24 & 2.39 \\
\hline${ }^{\mathbf{2 0 9}} \mathbf{B i}$ & 1606.16 & 5.55 & 1640.30 & 5.52 \\
\hline
\end{tabular}

The weakly-bound projectile ${ }^{6} \mathrm{Li}$ is constructed by making use of the nucleon configurations of the stable $d$ and $\alpha$ nucleus generated as discussed earlier. The two nuclei are placed with respect to each other in a configuration corresponding to the potential energy between them being equal to the observed breakup energy (-1.467 $\mathrm{MeV}$ ). Similarly, ${ }^{7} \mathrm{Li}$ is constructed from $t$ and $\alpha$ configurations with the potential energy between them equal to the observed breakup energy $(-2.47 \mathrm{MeV})$. Ground-state properties of ${ }^{6} \mathrm{Li}$ and ${ }^{7} \mathrm{Li}$ generated by the above procedure are also given in the Table- 1 .

It may be noted that the ground-state properties of the generated nuclei in Table- 1 are not in close agreement with the experimental values. Therefore, we do not expect very close agreement for the calculated fusion cross sections also with the experiments. In spite of this problem, the model calculations however, do provide a reasonable qualitative description of the processes involved in fusion of weakly bound nuclei.

In order to maintain initially defined clusters, nucleon coordinates of the individual clusters are rigid-body constrained till they approach distances very close to the fusion barrier in the stage- 3 in the 3 S-CMD model simulation.

\subsection{Dynamical model for the collision trajectory}

The dynamical collision simulation is carried out in the $3 \mathrm{~S}-\mathrm{CMD}$ model $[10,11]$ which proceeds in the following three stages:

(1) Rutherford trajectory calculation: The projectile and the target nuclei are brought along their Rutherford trajectories with given $\mathrm{E}_{\mathrm{cm}}$ and impact parameter $(b)$ up to a very large initial separation $R_{\text {in }}=2500 \mathrm{fm}$.

(2) CRBD model calculation: The 3-body system of the target and the projectile, with its two constituent nuclei held rigidly, are placed on the Rutherford trajectories at $\mathrm{R}_{\text {in }}$ with their ground-state configuration of nucleon positions. This target-projectile system, as a 2-body system, is then allowed to evolve further using the CRBD-model calculation [9]. The motion of the centre of mass and the orientation of the principal axes of the target and projectile are obtained from the classical equations of motion for rigid bodies under the influence of the ion-ion potential and the torques generated by interaction between all the nucleons in the combined system.

(3) CMD calculation: The rigid-body condition on all the nuclei and the bond between the projectile fragments are relaxed at about $\mathrm{R}_{\mathrm{cm}} \approx 13 \mathrm{fm}$ which is very close to the barrier. The trajectories of all the participating nucleons are obtained by solving classical equations of motion:

$$
m \frac{d^{2} \boldsymbol{r}_{\boldsymbol{i}}}{d t^{2}}=-\nabla_{i}\left[\sum_{j \neq i} V_{i j}\right]
$$

for all the nucleons in a CMD-model approach [12]. If one or both the projectile fragments are further constrained to be rigid then these nuclei are dynamically evolved as in the CRBD calculation even in the stage- 3 .

\section{Dynamical simulations of ${ }^{6} \mathrm{Li}+{ }^{209} \mathrm{Bi}$}

In the present simulation of ${ }^{6} \mathrm{Li}+{ }^{209} \mathrm{Bi}$ collision, $\alpha$ and $d$ are treated as rigid bodies and the $(\alpha+d)$ cluster representing ${ }^{6} \mathrm{Li}$ is dynamically evolved as a rigid body till the target-projectile separation is close to their barrier top $(\sim 13 \mathrm{fm})$. At this separation the rigid-body constraint on the bond between $\alpha$ and $d$ is relaxed for further evolution of the entire system. The rigid-body constraint on ${ }^{209} \mathrm{Bi}$ is also relaxed at this stage.

Figure- 1 shows ${ }^{6} \mathrm{Li}+{ }^{209} \mathrm{Bi}$ collision at $\mathrm{E}_{\mathrm{cm}}=42.7 \mathrm{MeV}$ at different impact parameters $b$ resulting in: (i) complete fusion (CF) in figure-1(a), where the bond between $\alpha$ and $d$ remains intact and both are sticking to the target nucleus ${ }^{209} \mathrm{Bi}$; (ii) incomplete fusion (ICF) in figure-1(b), where $\alpha$ is captured after breakup from the projectile; (iii) scattering with projectile breakup (BU-Scatt.) in figure1(c) and; (iv) scattering without breakup in figure-1(d). Although not shown in this figure, we can also distinguish between DCF and SCF events [15]. In some cases $d$ is also broken leading to a process equivalent to stripping reaction with possible fusion of $\alpha$ with the target along with $n$ and scattering of $p$ [15]. This demonstrates the possibilities of simulations of all types of reaction mechanisms in a heavy-ion collision involving a weakly bound projectile. 


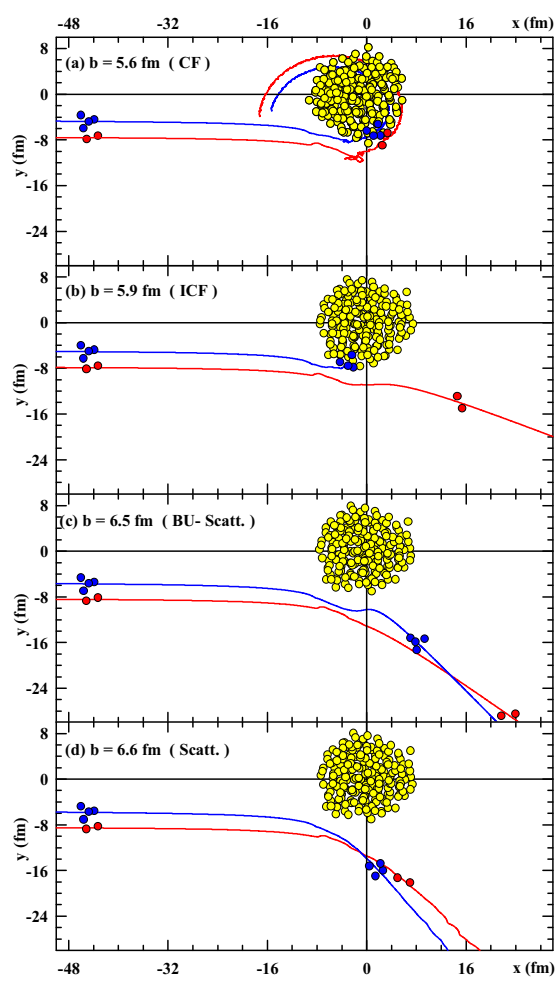

Figure 1: ${ }^{6} \mathrm{Li}+{ }^{209} \mathrm{Bi}$ collision at $\mathrm{E}_{\mathrm{cm}}=42.7 \mathrm{MeV}$ for different impact parameters b. Centre of mass trajectories of $\alpha$ and $d$ are shown by corresponding lines with their configuration of nucleons shown at two different times on the trajectories. Nucleon configuration of ${ }^{209} \mathrm{Bi}$ is shown at the later instant [14].

\section{Fusion cross sections}

In the CRBD calculations fusion is defined merely as a crossing of a fusion barrier. In the present calculations however, complete fusion (CF) is defined as an event in which both the projectile fragments are captured by the target nucleus for a sufficiently long interval of time, irrespective of whether they breakup (SCF) before being captured or, do not breakup (DCF).

Using the sharp cut-off approximation it is assumed that all the trajectories with $b<b_{\text {cr-CF, }}$ critical impact parameter for $\mathrm{CF}$, are fused and those for $b>b_{\mathrm{cr}-\mathrm{CF}}$ are scattered or lead to ICF events. Ion-ion potential is obtained as a function of the separation between the centre of masses of the target and the projectile fragments for $b=b_{\text {cr-CF. }}$.

For a given collision energy $\mathrm{E}_{\mathrm{cm}}$, a large number of randomly chosen (Monte-Carlo sampled) initial orientations (500 at higher energies and 2000 at lower energies) are considered and $b_{\text {cr-CF }}$ is determined for every orientation. Barrier parameters $\left(\mathrm{V}_{\mathrm{B}}, \mathrm{R}_{\mathrm{B}}, \omega_{\mathrm{B}}\right)$ obtained from the dynamically generated ion-ion potential corresponding to $b_{\text {cr-CF }}$ are used in the Wong's formula [16],

$$
\sigma_{f u s}=\left[\frac{\hbar \omega_{B}}{2 E_{c m}}\right] R_{B}^{2} \ln \left[1+\exp \left(2 \pi \frac{E_{c m}-V_{B}}{\hbar \omega_{B}}\right)\right]
$$

to calculate fusion cross section for a given orientation. An average over different orientations gives fusion cross section for a given collision energy.
Although, in the present calculations the trajectories and the barrier parameters are obtained classically but quantum tunneling is taken into account, by using the above formula in a semi-classical manner.

\section{$4.1{ }^{6} \mathrm{Li}+{ }^{209} \mathrm{Bi}$ collision}

We calculate $\mathrm{CF}$ cross sections for ${ }^{6} \mathrm{Li}+{ }^{209} \mathrm{Bi}$ reaction for various cases by systematically relaxing the rigid-body constraints on the target, the bond between the projectilefragments, and the projectile fragments itself, viz:

(a) ${ }^{6} \mathrm{Li}$ and ${ }^{209} \mathrm{Bi}$ are treated as rigid-bodies (CRBD calculation);

(b) Both $\alpha$ and $d$ are treated as rigid-bodies in ${ }^{6} \mathrm{Li}$ but are free to move with respect to each other for $\mathrm{R}_{\mathrm{cm}}<13 \mathrm{fm}$; (c) Same as in (b) but allowing $d$ also to become nonrigid in stage- 3 and possibly breakup.

Target ${ }^{209} \mathrm{Bi}$ is non-rigid in the case (b) and (c) in stage- 3 of the $3 \mathrm{~S}-\mathrm{CMD}$ model. Calculated $\mathrm{CF}$ cross sections for ${ }^{6} \mathrm{Li}+{ }^{209} \mathrm{Bi}$ reaction for the above cases are shown in figure-2.

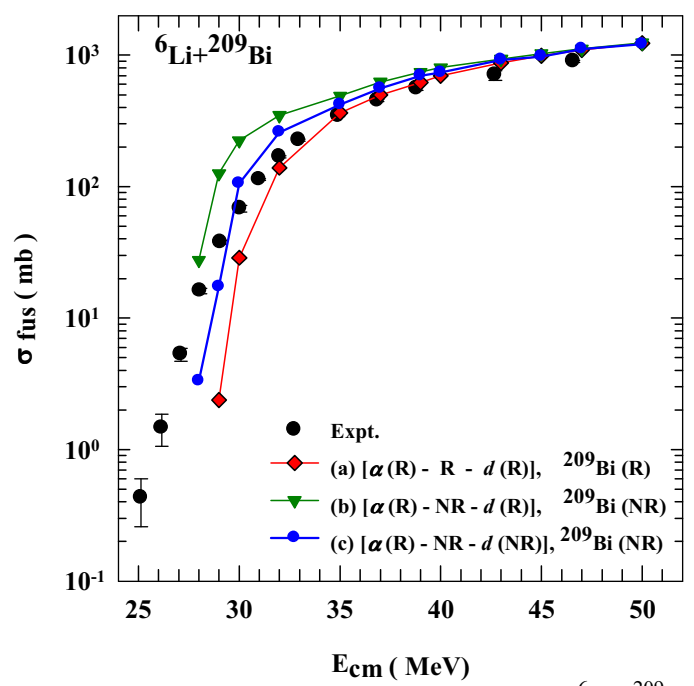

Figure 2: Complete fusion cross sections for ${ }^{6} \mathrm{Li}+{ }^{209} \mathrm{Bi}$ system for the cases (a), (b), and (c). (R: rigid, NR: Non-rigid).

In the case-(a), since there is complete lack of internal excitations in the rigid projectile and the target, which tends to lower fusion probability [11], hence the calculated CF cross sections are highly underestimated at lower energies as compared to that for the case-(b) as well as the experimental data [2].

In the case-(b), although the two fragments being rigid do not have internal excitations, the projectile itself is excited as the bond between the fragments is relaxed. This even leads to projectile break up, resulting in loss of flux from $\mathrm{CF}$ events. This contributes to breakup events like ICF which increases as the collision energy is increased [15]. Therefore, we find that the otherwise possible enhancement of CF cross section in the case-(b) due to the projectile excitations is suppressed at higher energies resulting in similar values as in the case-(a).

Since the $d$, in the projectile fragment, also has very low binding energy which can lead to its own breakup. Therefore, we consider the case-(c) in which breakup of $d$ 
results, additionally, in a process which is similar to $n$-stripping reaction followed by breakup of the resultant unstable ${ }^{5} \mathrm{Li} \rightarrow \alpha+p$ leading to ICF events, with $(\alpha+n)$ capture equivalent to ${ }^{5} \mathrm{He}$ capture events [4, 15]. Once again this results in compensation of the possible enhancement due to excitations, compared to case-(a) at higher energies and also leads to a significant reduction in CF cross sections compared to the calculation in which $d$ is also kept rigid in case-(b). Comparison of the CF cross section for case-(c) with the experiment shows reasonable agreement above $\mathrm{E}_{\mathrm{cm}}=29 \mathrm{MeV}$ data.

\section{$4.2^{7} \mathrm{Li}+{ }^{209} \mathrm{Bi}$ collision}

For ${ }^{7} \mathrm{Li}+{ }^{209} \mathrm{Bi}$ reaction we have calculated $\mathrm{CF}$ cross section by finding the barrier parameters for head-on collision $(b=0)$ only. The CF cross sections are calculated for the three cases as in ${ }^{6} \mathrm{Li}+{ }^{209} \mathrm{Bi}$ reaction, viz:

(a) ${ }^{7} \mathrm{Li}$ and ${ }^{209} \mathrm{Bi}$ treated as rigid-bodies;

(b) Both $\alpha$ and $t$ treated as rigid-bodies in ${ }^{7} \mathrm{Li}$ but free to move with respect to each other for $\mathrm{R}_{\mathrm{cm}}<13 \mathrm{fm}$;

(c) Same as in (b) but allowing $t$ also to become non-rigid in stage-3 with its possible breakup.

Target ${ }^{209} \mathrm{Bi}$ is non-rigid in the case (b) and (c) in stage- 3 . Calculated $\mathrm{CF}$ cross sections for ${ }^{7} \mathrm{Li}+{ }^{209} \mathrm{Bi}$ reaction for the above cases are shown in figure- 3 .

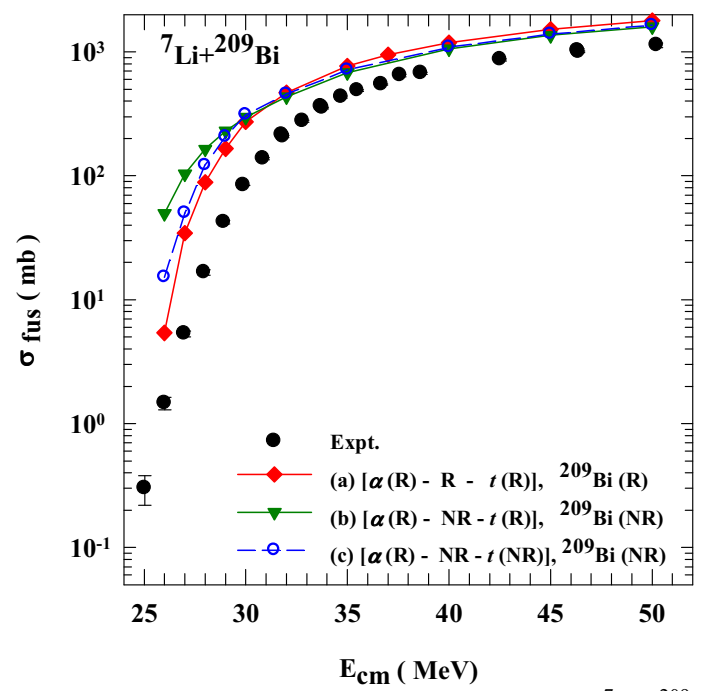

Figure 3: Complete fusion cross sections for ${ }^{7} \mathrm{Li}+{ }^{209} \mathrm{Bi}$ system for the cases (a), (b), and (c). (R: rigid, NR: non-rigid).

Calculated results for the three cases for ${ }^{7} \mathrm{Li}+{ }^{209} \mathrm{Bi}$ reaction show qualitatively similar behavior as discussed in the case of ${ }^{6} \mathrm{Li}+{ }^{209} \mathrm{Bi}$ reaction, but with differing magnitudes of differences between them as compared to that between the cases in ${ }^{6} \mathrm{Li}+{ }^{209} \mathrm{Bi}$ reaction due to the differences in the breakup energies. CF cross sections for all the cases are overestimated compared to the experimental data for ${ }^{7} \mathrm{Li}+{ }^{209} \mathrm{Bi}$ reaction [2]. Large overestimation even in the case-(c) of $\alpha$ being rigid and $t$ non-rigid, however, indicates lack of some other possible mechanisms in the present calculations which might be responsible for the observed fusion suppression; notably, 1- $p$ pickup by $t$ in ${ }^{7} \mathrm{Li}$ and then breaking up of the resulting ${ }^{8} \mathrm{Be}$ into two $\alpha$ channel, as observed in a recent experiment [4].

\section{Conclusions}

The model presented here incorporates the reorientation effects on the deformed target/projectile and their excitations at close distances. The essential features of breakup reactions such as complete fusion, incomplete fusion, scattering with and without breakup are also demonstrated in the model calculations.

The comparative study of calculated CF cross sections for ${ }^{6} \mathrm{Li}+{ }^{209} \mathrm{Bi}$ and ${ }^{7} \mathrm{Li}+{ }^{209} \mathrm{Bi}$ reactions with systematic relaxations of the rigid-body constraints on the target, projectile fragments and the bond between the projectile fragments exhibits the importance of constituent's excitations and breakup. While as a result of allowing for internal excitations, CF cross sections are comparatively enhanced, breakup of the constituents takes away the flux from the $\mathrm{CF}$, resulting in its comparative suppression.

The CF cross section calculation which allows for the breakup of ${ }^{6} \mathrm{Li}$ into $\alpha$ and $d$ as well as breakup of $d$ itself, gives reasonable agreement with the experiment which is also in conformity with the recent experimental observation of the importance of direct reaction processes as well. On the other hand, in the case of ${ }^{7} \mathrm{Li}+{ }^{209} \mathrm{Bi}$ reaction, similarly allowing for the breakup of ${ }^{7} \mathrm{Li}$ into $\alpha$ and $t$, as well as allowing for the breakup of $t$ itself is, not sufficient to give good agreement with the experiment in the present model calculations, which indicates lack of some other mechanisms in the model calculations.

\section{Acknowledgment}

This work was carried out with the financial assistance under a DAE-BRNS project no. 2009/37/20/BRNS.

\section{References}

1. M. Dasgupta et al, Phys Rev C 66, 041602 (R) (2002)

2. M. Dasgupta et al, Phys. Rev. C 70, 24606 (2004)

3. L. F. Canto et al, Phys. Rep. 424, 1 (2006)

4. D H Luong et al, Phys Rev C 88, 034609 (2013)

5. K. Hagino et al, Phys. Rev. C 61, 037602 (2000)

6. H. D. Marta et al, Phy. Rev. C 89, 034625 (2014)

7. A. Diaz-Torres et al, Phy. Rev. Lett. 98, 152701 (2007)

8. C. K. Phookan, K. Kalita, J. Phys. G 40, 125107 (2013)

9. P. R. Desai, S. S. Godre, Eur. Phys. J. A 47, 146 (2011)

10. M. R. Morker, S. S. Godre, Proc. Symp. on Nucl. Phys., 57, 560 (2012)

11. S. S. Godre, this proceedings (to be published)

12. S. S. Godre, Y. R. Waghmare, Phys. Rev. C 36, 1632 (1987)

13. W. D. Myers, W. J. Swiatecki, Ann. Phys. (N.Y.) 55, 395 (1969)

14. M. R. Morker, S. S. Godre, Proc. Symp. on Nucl. Phys., 56, 644 (2011)

15. M. R. Morker, S. S. Godre, Proc. Symp. on Nucl. Phys. (to be published) (2014).

16. C. Y. Wong, Phys. Rev. Lett., 31, 766 (1973) 